\title{
¿Lo cuálo? Una "nueva” partícula (re)funcionalizada en español actual ${ }^{*}$
}

\author{
¿Lo cuálo? A "new" (re)functionalized particle in modern Spanish
}

\author{
ENRIQUe PATo \\ Université de Montréal \\ enrique.pato-maldonado@umontreal.ca \\ ORCID iD: 0000-0002-6955-286I
}

Recibíu / Received: 24-VII-20 I 8

Aceptáu / Accepted: I I-III-20I9

RESUMEN. El presente trabajo se focaliza en el estudio del interrogativo ¿lo cuálo?, forma subestándar del español coloquial, presente asimismo en otras lenguas. En cuanto a su extensión geográfica, la revisión de los datos del Corpus del español: Web/Dialectos muestra que es un rasgo propio del español europeo, aunque también se puede registrar esporádicamente en algunos países de América (México y Argentina). La documentación histórica y actual de esta forma vernácula nos indica que el uso interrogativo ha sido y es el más abundante (frente al uso como relativo). La descripción de ¿lo cuálo? como partícula (re)funcionalizada se centra en sus funciones, su prosodia, su posición y su sintaxis. Por último, la revisión del proceso de (re)funcionalización de esta partícula se dirige a la creación de una nueva construcción a partir de una ya existente (lo cuál) para transmitir un valor similar al de cómo y (el) qué, que se deduce del contexto (interrogativo) en el que aparece, como propiedad semántica inherente. La "nueva" partícula se emplea ahora con otros fines comunicativos: irónicos y jocosos.

Palabras clave: español, gramática, variación, funcionalización, lo cuálo.

ABSTRACT. Present work focuses on the study of the interrogative ¿lo cuálo?, a substandard form in colloquial Spanish, also present in other languages. Regarding its geographical extension, the review of Corpus del español: Web/Dialects data shows that it is a characteristic phenomenon of European Spanish, although it can also be recorded sporadically in some varieties in American Spanish (Mexico and Argentina). The historical and current documentation of this vernacular form indicates that the interrogative use has been and still is the most abundant (compared to its use as relative pronoun). The description of ilo cuálo? as a (re)functionalized particle focuses on its functions, its prosody, its position, and its syntax. Finally, the review of the process of functionalization of this "new" particle concentrates on the creation of a new construction from an existing one (lo cuál) to transmit a similar value to cómo y (el) qué, that it is deduced from

* El presente trabajo ha sido financiado parcialmente por el Conseil de recherches en sciences humaines $(\mathrm{CRSH}) \mathrm{de}$ Canadá (proyecto 435-20 1 8-0526). Mi sincero agradecimiento a los dos revisores anónimos, por la lectura que realizaron del manuscrito preliminar y por sus atinados comentarios, que hicieron modificar la primera hipótesis de partida. Mi agradecimiento asimismo al Consejo de Redacción de la revista, por su estimable ayuda durante todo el proceso de publicación. 
the context (interrogative) in which it appears, as inherent semantic property. The "new" category is now used for other communicative purposes: ironic and jocular.

Keywords: Spanish, grammar, variation, functionalization, lo cuálo.

Cómo citar / Form of Citation: Pato, Enrique (2019): “¿Lo cuálo? Una 'nueva’ partícula (re)funcionalizada en español actual”, Glosema, I.2019.177-I94. https://doi.org/I0.178 I I/glosema.I.2019.177-194.

\section{INTRODUCCIÓN Y OBJETIVOS}

Omo es sabido, y a diferencia de otras lenguas peninsulares como el asturiano (ALLA 200 : 232) —donde hay distinción de género (cuál-cuálu/cuálalcuálo) y de número (cuálos/cuáles)—, en español general normativo el interrogativo cuál (y el pronombre relativo cual) solo se flexiona en número (cuáles). Sin embargo, tal y como recuerda la Nueva gramática de la lengua española, "en algunas variedades del español rural" se documentan las formas femeninas cuala/cuála, cualas/cuálas y el neutro cualo/cuálo (RAE y ASALE 2009: I 559). Según la norma académica, este uso - marcadamente popular- debe evitarse en la lengua culta (RAE y ASALE 2005: I9I). A pesar de ser considerado un vulgarismo (forma estigmatizada) y estar condenado por la Academia (forma subestándar), el empleo de cualo/cuala se extiende por todo el territorio peninsular (Zamora Vicente i 967: 255) ${ }^{1}$, así como por algunos países americanos, tal y como se mostrará en este trabajo $(\$ 2)$.

Varios autores (Borao I884, Nogués I898: 64, Tomey I901: 27, Alvar I953, Nagore Laín I989, Vespertino Rodríguez 2002-2004: I744, Metzeltin 2009: I 54, entre otros) han seńalado que el relativo cualo, su forma compuesta el cualo y el interrogativo cuálo serían aragonesismos en castellano, ya que son formas propias del aragonés que se registran ampliamente en el Campo de Jaca (Alvar i 950: 93, Alvar y Pottier I983), Huesca (Selfa Sastre 2005-2006: I76), Luna (Marín i Ramos 2009: I7) y la comarca Gúdar en Teruel (López Navarrete y Torres Belmonte 2008: 49), entre otros lugares ${ }^{2}$. Lo interesante es que las formas (lo) cualo/cuálo se han extendido

\footnotetext{
${ }^{1}$ Desafortunadamente, los cuestionarios de los atlas lingüísticos, como el Atlas Lingüistico de la Península Ibérica ( $A L P I$ I 962), no contemplan el estudio de este pronombre, por lo que resulta complicado conocer su distribución geográfica en espańol europeo. Los atlas lingüísticos hispanoamericanos tampoco han incluido esta forma en sus cuestionarios. Los datos del Corpus oraly sonoro del español rural [COSER] (Fernández-Ordóńez 2005-2019), especialmente en las encuestas transcritas hasta mayo de 2018 , registran estas formas en las provincias de Zaragoza (Mesones de Isuela, $C O$ SER-47 I4, Mujer, 68 años), Cuenca (Cardenete, COSER-ı607, M, 70; Villaconejos de Trabaque, COSER-i636, H, 80), noroeste de Valencia (Mas de los Mudos, COSER-430 , H, 74), Murcia (Palacios Blancos, COSER-3 I I I, M, 80) y Almería (Lucainena de las Torres, COSER-04I 8, M, 85).

${ }^{2}$ Saroïhandy ( 1898 y I 90 I), por ejemplo, lo documentó en Graus y en los pueblos vecinos del Valle del Cinca (Ansó, Echo, Bielsa y Benasque). En lo que concierne a la morfología, el aragonés tiende a distinguir el género en muchos adjetivos y sustantivos terminados en - $e$ o consonante, como sucede en berda, dolenta, trista, granda, descreyenta, tristos y comunas (Umphrey I987 [I9II]: I86); fenómeno que ha sido registrado asimismo en textos aljamiados aragoneses en voces como amanta, yerra o çençerra (Vespertino Rodríguez 2002-2004: 1743). Este género analógico, como la formación de los masculinos rano (de rana) y cabro (de cabra) (Gómez Sánchez 20 I I: 234), es un procedimiento también muy vivo
} 
a otras regiones al sur de Aragón, como Murcia (Gómez Ortín 2004: 19) y Albacete (Gómez Sánchez 20I I: 234); sin olvidar que son formas propias en judeoespañol clásico (García Moreno 2004: 229) y moderno (Wagner I9I4, Zamora Vicente I967: 36 I, Díaz-Mas 1983: 235, Quintana 200 I: 177), del aragonés de los moriscos (Corominas y Pascual I980-I99I, vol. 2: 257), y, como indicábamos anteriormente, forman parte de la serie de pronombres interrogativos de la lengua asturiana (Alvar y Pottier 1983, Díaz González i 986: 50, ALLA 2001)³.

Por otro lado, en español coloquial ${ }^{4}$, sobre todo en su variante escrita, se documenta un "nuevo" uso del interrogativo (lo) cuálo como partícula funcionalizada (¿lo cuálo?), que todavía no ha recibido la atención suficiente en los trabajos previos de gramática descriptiva (RAE y ASALE 2009), ni en los trabajos sobre los marcadores del discurso y las partículas discursivas, por lo que se desconoce su comportamiento gramatical, semántico y pragmático. Dado que se trata de una estructura hasta cierto punto productiva en español actual, interesa describir esta partícula y conocer los contextos en los que su uso es relevante.

Teniendo en cuenta tres objetivos generales, el presente trabajo queda estructurado de la siguiente manera. Tras esta breve introducción y contextualización $(\$ \mathrm{I})$, se presenta la metodología, la descripción de los corpus empleados en la investigación y los primeros resultados obtenidos $(\$ 2)$. A continuación, se ofrece documentación abundante sobre estas formas vernáculas interrogativas (y relativas) en la historia de la lengua española $\left(\$_{3}\right)$, para cumplir con el primer objetivo. Después, en el apartado $\$ 4$, se emprende una primera descripción de la partícula funcionalizada lo cuálo, en relación a sus funciones, prosodia, posición y sintaxis, segundo de los objetivos de este trabajo. Por último, y para llevar a efecto el tercero de los objetivos, se ofrece un análisis del proceso de funcionalización por el que ha podido pasar esta “nueva” partícula $(\$ 5)$. El trabajo se cierra con unas conclusiones finales $(\$ 6)$.

\section{Metodología y PRIMERos RESUltados}

Para poder obtener datos suficientes, y que estos sean relevantes a la hora de ser descritos y analizados, sobre un fenómeno estigmatizado como el que nos ocupa en

\footnotetext{
en judeoespañol, con la creación de formas femeninas como jóvena (de joven) y capitala (de capital) (Núñez Méndez 2015: 108).

${ }^{3}$ Como sustantivo, el masculino tiene dos formas equivalentes (cuál y cuálu), pero como adjetivo antepuesto al sustantivo solo se usa una (cuál) (cf. ALLA 200I). El interrogativo neutro (cuálo 'qué cosa') es usual en el habla corriente, pero en asturiano no se emplea lo cualo (comunicación personal de Ramón de Andrés Díaz, Universidad de Oviedo).

${ }^{4}$ Como es sabido, la conversación coloquial se caracteriza por su menor grado de planificación, en comparación con la conversación formal. Además, suele haber una relación de proximidad mayor entre hablante y oyente y un saber compartido (Briz I998).
} 
este trabajo, es necesario contar con corpus actuales que permitan búsquedas automáticas en una gran cantidad de fuentes. Uno de esos corpus, y el primero que empleamos en esta investigación, es el Corpus del español: Web/Dialectos (Davies 2016), base de datos que cuenta con más de dos mil cien millones de palabras ${ }^{5}$ procedentes de más de dos millones de páginas web (prensa escrita, comentarios y blogs) de los 2 I países de habla hispana, publicadas entre 2013 y 20I4. A pesar de su inmenso contenido, este corpus recupera solo 52 casos de ¿lo cuálo?, tal y como se resume en el siguiente cuadro, por países, número de casos y porcentajes (\%).

\begin{tabular}{r|c|c}
\multicolumn{1}{c|}{ PaÍs } & CAsos & $\%$ \\
\hline España & 38 & 73.07 \\
\hline México & 4 & 7.69 \\
\hline Argentina & 3 & 5.76 \\
\hline Uruguay & 1 & 1.92 \\
\hline Perú & 1 & 1.92 \\
\hline Ecuador & 1 & 1.92 \\
\hline Colombia & 1 & 1.92 \\
\hline Nicaragua & 1 & 1.92 \\
\hline Cuba & 1 & 1.92 \\
\hline Rep. Dominicana & 1 & 1.92 \\
\hline Totales & 52 & 100
\end{tabular}

Cuadro i. Casos y porcentajes de lo cuálo en el Corpus del español

Los datos del cuadro precedente muestran claramente que estamos ante un fenómeno propio del espańol europeo, con un $73.07 \%$ (38 casos de los 52 obtenidos). Sin embargo, de manera esporádica, también se puede registrar en algunos países americanos de habla hispana, especialmente en México (4 casos de 52, $7.69 \%$ ) y en Argentina (3 casos de 52, $5.76 \%$ ).

El segundo de los corpus que empleamos en este trabajo en realidad no es un corpus lingüístico como tal, sino una red social que permite hacer búsquedas lingüísticas concretas gracias a su buscador. Se trata de Twitter. Numerosos autores

\footnotetext{
${ }^{5}$ En concreto, para las variedades del español de América el corpus contiene I 64I 448407 palabras y para las variedades del espańol europeo 4593 I 282 I palabras. Los porcentajes por áreas lingüísticas y países están equilibrados y corresponden con el peso demográfico de cada una de ellas.
} 
han empleado previamente, y con éxito, esta red social como fuente de datos lingüísticos ( $c f$., entre otros, Pato y de Benito 20 I7). Para poder controlar los resultados (número de casos y relevancia) hemos limitado la búsqueda de (lo) cuálo a unas fechas concretas, entre el 8 de enero y el 8 de abril de 2018 , lo que equivale a 3 meses de actividad de los usuarios. Los datos obtenidos desde nuestro servidor de Montreal se presentan a continuación, en el cuadro 2, por tipo de forma, número de casos y porcentajes (\%).

\begin{tabular}{r|c|c}
\multicolumn{1}{l|}{ FORMA } & CASOS & $\%$ \\
\hline Interrogativo & 826 & 95.71 \\
\hline Relativo & 30 & 3.48 \\
\hline Fraseología & 7 & 0.81 \\
\hline TOTALES & 863 & 100
\end{tabular}

Cuadro 2. Casos y porcentajes de lo cuálo en Twitter

De todos los casos recuperados en ese periodo limitado de tiempo (3 meses), (lo) cuálo aparece como pronombre interrogativo en un 95.7I \% (826 casos de 863), como pronombre relativo en un $3.48 \%$ ( 30 casos de 863 ) y en uso fraseológico tan solo en un $0.8 \mathrm{I} \%$ (7 casos de 863).

Los ejemplos de dicho lo cualo como los de (I $)^{6}$-englobados dentro de la categoría «fraseología» para los efectos de este trabajo-, que presentan un participio absoluto (dicho) y forman una estructura oracional con sujeto (RAE y ASALE 2009: $2088)^{7}$, no serán tratados en este trabajo:

(I) a. Pues ahora que lo dices choca, eh?? Es cierto que he utilizado palabras malsonantes para darle más dramatismo. "Dicho lo cualo" y pidiendo excusas sustituye por agallas/ cobardes y contesta si te parece. Gracias. (Twitter, 27/3/2018, ToniOliveras_, España)

b. Yo de ti le daba a unfollow a ese diario... Science CAN explain why. Dicho lo cualo, que te vaya bien el examen. Nos vemos a las sam en la sala de estudios. (Twitter, 20/3/2018, cptangerine, España)

También dejamos para un próximo trabajo el análisis de los casos en los que (lo) cualo actúa como pronombre relativo, como en los ejemplos de (2).

\footnotetext{
${ }^{6}$ En los ejemplos que ofrezco de la red social Twitter se indica la fuente, la fecha, el nombre de usuario, la ciudad/provincia y el país del mismo, siempre y cuando esta información esté disponible en su perfil. Los datos se presentan tal y como figuran originalmente, solo incluimos alguna coma (,) o punto y seguido (.) para facilitar la lectura; el resto del texto no ha sido corregido ni modificado. Para los casos del Corpus del español: Web/Dialectos indicamos solamente el país del usuario. Para los ejemplos literarios de $₫ 3$ doy el título de la obra, el autor de la misma, la fecha de publicación y, cuando es necesario, la ciudad o región de nacimiento del autor.

${ }^{7}$ Puede, asimismo, actuar como conector discursivo adverbial (RAE y ASALE 2009: 2360).
} 


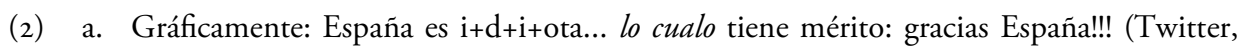
Io/3/2018, pilargayoso, España)

b. Todavía queda gente que te lee por aqui matuerza. Si estas en esa situación ambas opciones son y no son la correcta. Una apela a tu orgullo lo cualo me decantaría por ella pero en un tono serio sin llegar a ser despectivo. Pero esto es lo de siempre... (Twitter, 26/2/2018, JuanJosPallars, Espańa)

Por tanto, en esta ocasión, tal y como quedó indicado en $₫ \mathrm{I}$, nos detendremos en el estudio de (lo) cuálo como pronombre interrogativo y partícula funcionalizada. De los 826 casos registrados (v. el cuadro 2), lo cuálo puede aparecer solo, es decir de manera independiente en el discurso, en un 59.8 I \% (494 casos de 826), como se ejemplifica en (3a-b), o bien estar integrado en el discurso y formar parte de la oración correspondiente, en un $40.19 \%$ (332 casos de 826), como vemos en los ejemplos de (3c-d):

(3) a. Futbol? Lo cualo? Quien ve el futbol pudiendo verte a ti ayayay. (Twitter, 6/3/2018, Norah_5, Cataluña, España)

b. -Te gustan los animales? +Mucho. -Te gustan los videojuegos? +Mucho. - Te gusta trabajar? -Lo cualo?? (Twitter, 2/2/2018, Desatendido, Madrid, España)

c. A ver, ¿algún "técnico" munícipe que me explique lo cuálo es éso de "Cultura comunitaria”? Gracias, de nada. Regolución [sic] o muerte. (Twitter, I8/3/2018, manicoregolvedo, Aragón, España)

d. Que tú has pagado lo cuálo? Au! Va! Ve a robar a otro lado. (Twitter, I7/3/2018, MontseTsarnaeva, Cataluña, España)

Por último, para ofrecer documentación histórica relevante de las formas interrogativas (y relativas) en estudio (primer objetivo del trabajo), he revisado, además, las bases de datos del Corpus diacrónico del español (CORDE) y del Corpus de referencia del español actual (CREA). Estos ejemplos se presentan en el siguiente apartado.

\section{Documentación de las fORmas}

A lo largo de la historia de la lengua española la necesidad de establecer la concordancia de género y número entre cual y su antecedente ha sido constante, como intentaremos mostrar en este apartado. Esta forma vernácula, al igual que la estándar invariable, se comporta como pronombre relativo (v. 4a-b, los qualos) o interrogativo (v. 4c, cualas y $4 \mathrm{~d}$, cualo), ambas formas con el valor actual de quelqué, tal y como muestran los ejemplos recogidos tanto en autores aragoneses (Vallés y Berlanga) como castellanos (Fernández de Madrigal y López Silva). Como hemos visto en los datos presentados en el cuadro 2, el empleo interrogativo es el más abundante en español, antiguo y moderno: 
(4) a. Los primeros, que llamavan dioses escogidos, son veinte $\&$ dos, los qualos Seneca pone en algunos logares de sus obras et tracta de ellos mucho sant Agostin. (Libro de las paradojas, Alonso Fernández de Madrigal, I 438-I 445, Ávila)

b. Entre los buenos amigos no ay necesidad de toda esta diligencia. Porque ora esten presentes. Ora ausentes siempre en ellos ay verdadera amicicia. Verdad es Que los dos Refranes pueden tener otro enues y es que con el Ausenca de vno suelense mudar los amigos: los qualos estando presente eran fieles y estando ausente no lo son. (Libro de refranes y sentencias, Pedro Vallés, I 549, Aragón)

c. SEÑOR CANDELAS: le "azvierto" a "usté", y se lo "azvierto"/ muy seriamente que, o cambia/ de raíz... MARI-PEPA: ¡Señor Candelas! Señor Candelas: ...sus costumbres... MARIPEPA: Pero, ¿“cualas"? SEÑOR CANDELAS: ...o le pongo a "usté" los trastos/ en la calle. (La Revoltosa, José López Silva, I 897, Madrid)

d. Cuando le explicó que tendría "su-vención” (“mi ¿cualo?”, replicó el Alcalde), Francisco se quedó más tranquilo: -Así es la vida: hoy por unos, mañana por otros. (La gaznápira, Andrés Berlanga, 1984, Guadalajara)

Por otro lado, aunque su uso no es muy abundante, cualo también puede aparecer como relativo solo, como se comprueba en el siguiente ejemplo de Arniches. Este empleo no existe en otras lenguas peninsulares como el asturiano:

(5) Señor Sidonio Créeme a mí, deleite: unos chinchón, otros cazalla, otros monóvar, cualo valdepeñas, quién méntrida, nos diferenciaremos en el punto geográfico; pero, al remate, todos turcas. (El zapatero filósofo o año nuevo, vida nueva, Carlos Arniches, I917, Alicante)

Los ejemplos de (6), a continuación, presentan las formas cualos (v. 6a) y cualas (v. 6b) con artículo y antecedente expreso. En estos casos concretos, de principios del siglo XVI y procedentes de traducciones del francés, el valor que adquiere cualo es de adjetivo relativo antepuesto al sustantivo, uso literario muy poco frecuente incluso hoy en día con la forma estándar cual (Seco i986: I37-I 38, RAE y ASALE 2005: 19I). En realidad, el relativo funciona en estos ejemplos como un reasuntivo (subrayamos los nombres que se retoman tras el relativo de apoyo):

(6) a. Tenía, otrosí, las muy iguales e derechas espaldas, e los hermosos e bien apuestos braços, los cuales parescían no denegar los dulces abraços. E sus graciosas manos no eran ni punto villanas ni gruesas, cuyos dedos eran luengos e delgados. E las uńas parescían ser de marfil. Los cualos braços, manos e dedos parescían ser de color de nieve. (Tristán de Leonís, Juan de Burgos tr., I 50I)

b. Mas conujenete primera mente propensar el decorrimjento dela sangre $\&$ de quales venas sea fecho. Por que delas venas frecuran de poluos las qualas venas mueven del figado. (Tratado de cirugía de Tedrico, I 509)

Como pronombre interrogativo, puede aparecer precedido de la preposición de (v. $7 \mathrm{a}-\mathrm{b})$ y con valor deíctico discursivo (v. $7 \mathrm{c}$ ), tal y como veremos más adelante $(\$ 4)$. 
(7) a. Presidente de qué, de dónde, de cuálo?? De Chirona será Presidente y con honores!! (Twitter, 3/r/2018, AlvaroJuer, España)

b. ¿Comités de defensa de lo cualo...? ¡No son más que nazis destrozando la ley y el orden! (Twitter, 25/3/2018, supermegacholo, España)

c. -Do you speak english? -Lo cuálo? (Twitter, 22/I/20 18, lluisgaas, Cataluña, España)

Como quedó apuntado en $\$$ I, las formas femeninas (cuala, cualas) del pronombre interrogativo se registran especialmente en español actual. Los ejemplos de (8) muestran casos de cuala como interrogativo (v. 8a-b), precedido en ocasiones de la preposición por (v. 8c-d):

(8) a. Ok, ahora dime cuala es tu propuesta para salir de la dictadura, cuanto tiempo mas esperamos y como es la solución. (Twitter, 24/1/20 18, pedromerida, España)

b. Y cuala es la envidia de uds con un abogado, a él le pagan xa [para] defender a un acusado sea cual sea el caso. (Twitter, 7/I/2018, gelvezjota, España)

c. Quiero abrir una cuenta en una casa de apuestas y no se por cuala decidirme, vosotros que me aconsejais. (Twitter, 20/1/2018, antonioperegri2, Madrid, España)

d. En serio? Qué fuerte? Pero no entiendo por cuala razón lo hacen, no se puede tener amigos ya o que pasa? (Twitter, 6/r/2018, RushaNavarro, España)

Por último, también es posible documentar usos irónicos y festivos con las formas cualo, cuala, sobre todo en las fórmulas lexicalizadas cada cual o cada cuala (v. 9a), cada cual con cada cuala (v. 9b), cada cual con su cada cuala o su cada cualo (v. 9c) o tal para cualo (v. 9d), tanto en el español de España como en otros países americanos (Uruguay y Venezuela, en los ejemplos recuperados del Corpus del español: Web/Dialectos):

(9) a. de los gritos amatorios mejor no hablamos, porque cada cual o cada cuala cuenta la fiesta según le va. (Corpus del español: Web/Dialectos, España)

b. el público de Mérida se divirtió a lo grande y él se llevó a su casa un capazo de orejas y hasta un indulto, allá cada cual con cada cuala. (Corpus del español: Web/Dialectos, España)

c. es más divertido que tengamos algunas diferencias y que a esas diferencias las cuidemos sin incurrir en la homofobia. Cada cual con su cada cuala o su cada cualo. (Corpus del español: Web/Dialectos, Uruguay)

d. gracias al burro de Maduro y de Diosdado Cabello, son tal cual para cualo par de ladrones por eso el país está como está. (Corpus del español: Web/Dialectos, Venezuela)

\section{Descripción de la partícula ¿lo cuálo?}

Antes de presentar la descripción (valores y usos) de la que consideramos una nueva partícula funcionalizada en español actual, nos detendremos brevemente en 
dos aspectos: I) la imposibilidad de considerar en la actualidad ¿lo cuálo? como una partícula discursiva, y II) recordar los valores de lo cual en español estándar.

Siguiendo los trabajos realizados para el español de Briz (1998), Briz y Grupo Val.Es.Co (2008), Pons (1998), Portolés (1998) y Briz, Pons y Portolés (2008), la partícula discursiva es aquel elemento que sirve para guiar la interpretación del discurso, por lo que su función va más allá de la gramática, y suele ser, además, el resultado de un proceso de gramaticalización. Por otro lado, el valor de cada partícula discursiva está ligado directamente a la interacción entre el hablante y el oyente, por lo que es pragmática y discursiva. El caso concreto que nos ocupa (¿lo cuálo?) no puede ser tratado como una partícula discursiva porque no cumple con lo definido para este tipo de partículas. En efecto, aunque en ocasiones - como veremos en breve- puede guiar la interpretación del discurso, y puede funcionar como enunciado independiente, no comparte el resto de las características de las partículas discursivas: movilidad, formar grupo entonativo independiente y cambio significativo. Por otro lado, aunque su valor puede estar ligado a la interacción y podría ser el resultado de un proceso parcial de gramaticalización, tampoco presenta las funciones básicas de una partícula discursiva (Briz 2008: 2 I7): I) la conexión (argumentativa, reformuladora o estructuradora), II) la modalización de lo que se dice y del punto de vista del hablante, III) la focalización, al destacar un elemento expreso frente a una alternativa expresa o sobreentendida, y IV) el control del contacto, o la relación entre hablante y oyente. Además, su función no va más allá de la gramática, ya que se corresponde con la categoría gramatical del pronombre.

Por lo que respecta a lo cual, como es sabido, en español general se emplea cuando el antecedente es una oración (RAE y ASALE 2009: 1576$)^{8}$, por lo que sirve para recuperar parte de una predicación, toda una oración o incluso un conjunto de oraciones (visto lo cual; hecho lo cual; dicho lo cual). Este uso es propio de la deixis discursiva, en el sentido de que relaciona el miembro del discurso en el que se encuentra con el discurso anterior (Pérez Álvarez et al. 20 I4: 54). Además, casi siempre se emplea como referente de un elemento presente en el texto o en el contexto (esto es, de un conjunto conocido), nunca para referencias imprecisas o genéricas.

En relación con esto, y por lo que respecta al artículo neutro (lo), la naturaleza abstracta de su designación lo posibilita "para asociarse anafóricamente a contenidos de valor preposicional expresados con anterioridad en el discurso" (RAE y ASALE 2009: I082), como vemos en (IOa). No obstante, como pronombre interrogativo puede aparecer solo (¿cuálo?), como en (iob):

\footnotetext{
${ }^{8}$ Como relativo complejo, manifiesta en la sintaxis "los contenidos que pueden expresar otras piezas léxicas simples (quien, cuanto)" (RAE y ASALE 2009: 3294).
} 
(г) a. Amigas: llevar más de un estampado en la ropa queda MAL. Yo: lo cualo? A MI NO. (Twitter, I 5/2/20 1 8, amorasaco, Valencia, España)

b. de no haber sido porque podías poner juegos piratas en PSX y PS2 no se hubieran vendido ni una cuarta parte... - Cuálo??? No digas animaladas por Dios! La industria es lo que es hoy por hoy porque sencillamente se ha "gloabalizado" a todas las edades y géneros. (Corpus del español: Web/Dialectos, España)

Es suma, ¿lo cuálo? es una expresión interrogativa, similar a ¿qué? y ¿cómo?, pero de empleo en el registro escrito informal, menos normativo. El valor que toma es interrogativo o exclamativo, en el sentido de que se conforma como una petición de aclaración o una expresión de sorpresa.

Teniendo en cuenta modelos descriptivos previos (Briz, Pons y Portolés 2008), las funciones de lo cuálo pueden ser las siguientes: I) la conexión reformuladora parcial (v. I Ia), que deriva directamente del valor deíctico del pronombre; II) la modalización mediante intensificación (uso interrogativo y exclamativo) de lo que se dice (v. I rb); III) la focalización (o dislocación a la izquierda) para destacar un elemento expreso (v. I Ic-d); y IV) servir en el control del contacto entre hablante y oyente (v. I Id-e). Se emplea, en la mayoría de las ocasiones, para indicar sorpresa y sarcasmo:

(I I) a. Por simple comprobación, pedimos que verifiquen que mi línea tiene activada la tarifa de datos. Obviamente, la respuesta es no. ¿Lo cualo? Que no, que en 24 horas podría disponer de ello. (Corpus del español: Web/Dialectos, Nicaragua)

b. me pregunta si me parece guapa su hermana... ¿Lo cualo! La verdad es que tiene su encanto la chica. (Corpus del español: Web/Dialectos, Colombia)

c. Lo cualo??? Perdona, no me he enterado. Últimamente estoy más pendiente de los fachas catalanistas que de los fachas de Moncloa. (Twitter, 26/3/2018, PatiPatiBcn, Barcelona, España)

d. Lo cualo? Este tipejo repugnante dice que ya se ha explicado? Como pueden tener esa desfachatez y ese morro??? En otros tiempos ya hubieran perdido, literalmente, la cabeza. Como echo de menos esos tiempos. (Twitter, 23/3/2018, KarKari961, Bilbao, España)

e. - - Señora Cifuentes, tiene usted que aprobar las asignaturas antes de poder entregar el TFM. -Lo cualo?? (Twitter, 2 I/3/2018, Loba_Madre, Madrid, España)

En cuanto a su prosodia, la partícula lo cuálo se pronuncia con acento de intensidad en la /a/ de cuálo (recordemos que cual es el único relativo tónico del español). Presenta un contorno melódico propio, delimitado por anticadencia [ $\uparrow]$, lo que permite que pueda ser distinguido del resto de los elementos con los que se enlaza (con pausa anterior y posterior). El entorno prosódico se suele reflejar, en la mayoría de los ejemplos escritos, a través signos de interrogación, tal y como hemos visto en los ejemplos de (IO-II). 
Por lo que respecta a su posición, se puede situar tanto al inicio de su miembro del discurso (v. I 2a-b) como al final (v. I 2c-d). No se ha documentado, en cambio, en el interior del miembro del discurso.

(I2) a. Lo cualo? un PDF del Banco de España? más veracidad no sé... lo mismo quiere usted un documento de la ANC o de Omnium, no? Datosmacro, y La Vanguardia tampoco le valen? en cualquier caso con el BDE debería tener suficiente. (Twitter, 28/3/20I8, Flp_lts, España)

b. Lo cualo? Oye, en confianza... No has pensado en recibir ayuda profesional? Porque vives en un mundo paralelo a la realidad... Méditalo, te vendrá bien. (Twitter, 6/1/2018, spainwonderland, España)

c. Gregorio Serrano: "Nosotros tenemos preparado todos los medios materiales y humanos de los que disponemos para velar por la seguridad y fluidez de los desplazamientos". ¿Lo cualo? (Twitter, 29/3/20 I 8, Aratzeo, España)

d. Yo en Escocia: Me entiendo perfectamente, o problemas. Amiwi de todos. Yo en Southend: Lo cualo? (Twitter, 27/3/20 i 8, Ireneskyworld, España)

Con respecto a su sintaxis, el miembro del discurso en el que aparece es generalmente un sintagma no oracional, aunque con menor frecuencia también puede ser una oración (generalmente independiente). Aparece como respuesta a una pregunta previa o como reacción a una intervención anterior (v. I 3a-c). Por otro lado, aunque su uso no condiciona la selección modal, se emplea sobre todo en oraciones en modo indicativo (v. I 3c):

(I3) a. El transhumanismo es un movimiento cultural e intelectual, una forma de vida, una especulación afianzada en los avances tecnológicos, una visión científica que pretende conducir la evolución humana desde la Humanidad en la que nos encontramos a una Posthumanidad. ¿Lo cualo? (Twitter, 22/3/20 I 8, donisraelalonso, Cádiz, España)

b. ¡no confundas nunca el átomo de un elemento con la molécula de un elemento! ¿Lo cuálo? Podemos estar hablando de, por ejemplo, el átomo de hidrógeno (I electrón y I protón) o de la molécula de hidrógeno (dos átomos de hidrógeno unidos mediante un enlace covalente). (Corpus del español: Web/Dialectos, España)

c. Los mossos organizaron lo cualo? si les ofrecieron salir por la azotea y pasar al edificio de al lado... venga va... que lo vimos todos. (Twitter, 30/3/2018, Ciutada, Barcelona, España)

Hasta ahora hemos mostrado que lo cuálo está marcado - por su mayor frecuencia de uso- en la lengua escrita y, en concreto, en el registro coloquial. Sin embargo, puede aparecer también en el discurso formal e intermedio — entre formal e informal- (v., por ejemplo, izb).

Además, como vimos en los ejemplos de (I), lo cualo también puede formar parte de algunas combinaciones rutinarias con valor conectivo (construcciones absolutas). En concreto, los casos de (I 4a-b) muestran cómo lo cualo se sitúa al inicio de la 
oración y recupera la información anterior, como posible comentario digresivo, ejemplos que hemos nombrado como fraseología:

(I4) a. Ojalá recupere el nivel de sus primeros x-men. $Y$ dicho lo cualo, yo creo que Predicador es una obra que jamás debería adaptarse. (Corpus del español: Web/Dialectos, España)

b. de tontada en tontada a cargo del presupuesto público. A pesar de lo cualo se la recibió en su momento como posible futura candidata a la Secretaría General del Partido Socialista... (Corpus del español: Web/Dialectos, Cuba)

En lo que concierne a su proceso parcial de gramaticalización, como veremos en el siguiente apartado $(\$ 5)$, el camino seguido ha sido de pronombre a partícula funcionalizada. Como pronombre relativo (formas masculina cualo y femenina cuala) tiene el significado de 'como, de la misma manera que'; como adverbio relativo culto, el de 'como' ('en el modo o la manera que'); y como adverbio, el de 'así como' (denota además comparación). Por todo ello, la partícula ¿lo cuálo? es semánticamente similar a ¿cómo?

\section{Sobre el PROCeSo DE (RE)FUnCiONALizaCión DE ¿lo cuálo?}

Como hemos visto en los ejemplos precedentes (especialmente IO-I3), para el procesamiento discursivo de las construcciones con lo cuálo se requiere un conocimiento previo de la situación. Entre las variables que determinan el entorno del discurso se han señalado las siguientes: organización del texto, fuente de información, actitudes del hablante (subjetividad), interacciones del hablante-oyente (intersubjetividad), situación del discurso y conocimiento del mundo ( $c f$., por ejemplo, Kaltenböck, Heine y Kuteva 20I I).

Así las cosas, creemos que el fenómeno en estudio puede explicarse mejor gracias a la teoría de la gramaticalización. Como ha sido establecido en este marco teórico, el material gramatical opaco, aquel que no puede entenderse espontáneamente, puede reutilizarse en algunas variedades (en nuestro caso, sobre todo en las variedades del español peninsular) para nuevos fines, como fenómeno más del discurso narrativo (cf. Van Dijk y Kintsch 1983). Este nuevo uso y significado (¿cuál? > ¿lo cuálo? '¿cómo, qué (cosa)?') depende del contexto real, el cual debe ser lo suficientemente pertinente como para desencadenar diversas inferencias semánticas o razonamientos deductivos en el destinatario, por lo que puede presentar un valor discursivo-pragmático nuevo ( ${ }_{i}^{*}$ lo cuáll?). 
En concreto, la refuncionalización (o exaptación) es un tipo específico de adaptación (Heine 2003, Norde 2009) ${ }^{9}$. Muchos casos de refuncionalización muestran procesos donde un elemento gramatical, que está a punto de desaparecer, adquiere un nuevo significado y finalmente se reubica dentro del sistema gramatical para expresar otro valor u otra función. La funcionalización (o capitalización), por su parte, es la adición de un nuevo valor al significado o función ya existente en un elemento. Ambos procesos, (re)funcionalización, nos ayudan a comprender mejor cómo se forma y cómo se mantiene el material gramatical dentro de las lenguas naturales.

En estos procesos - siempre dentro del marco de la gramaticalización (Harris y Campbell 1995, Willis 2010, entre otros) - los hablantes crean un nuevo modelo gramatical; en el caso que nos ocupa una nueva partícula (¿lo cuálo?). Esta nueva construcción sirve para transmitir un nuevo valor, similar al de ¿cómo? y ¿(el) qué (cosa)? enfatizado ${ }^{10}$. Las formas «lo + cuálo mantienen su significado básico, esto es, se reconocen como artículo y pronombre interrogativo; sin embargo, el valor de la construcción se deduce ahora del contexto discursivo específico en el que aparece (v. los ejemplos de I I-I 2 y de I 5), por lo que el lugar de este cambio gramatical sería el contexto. Estamos, por tanto, ante un cambio provocado por el uso contextual específico:

( I 5) a. Me acuerdo que lo vi en casa de un colega... me dijo has visto esto de iutuve? Y yo... lo cualo? Mira donde ha llegado la plataforma. De un dia en el zoo a llenarse de animales gobernados por monos. (Twitter, 21/4/2018, JordiForty, España)

b. Juez Llarena: de cuanto patrimonio dispone? Mireia Boya: lo cualo? Juez Llarena: libertad sin cargos. (Twitter, I4/2/2018,_Prebot, España)

Por otro lado, la (re)funcionalización puede tener lugar también cuando una forma gramatical pierde casi todo su contenido semántico original y se emplea como una forma semánticamente distinta ('reanálisis de la función' a la Heine 2003), es decir, casos en los que el significado de una construcción se desplaza a un contexto específico (el interrogativo en el caso de ¿lo cuálo?). Ocurre también cuando el elemento en cuestión no tiene una función o cuando se considera "basura lingüística", aunque no necesariamente tiene que ser "basura" (Lass I 990). Por último, el objetivo de la (re)funcionalización también puede ser una categoría ya existente (Traugott 2004). Estos tres procesos se podrían aplicar al caso de ¿lo cuálo?, ya que esta partícula

\footnotetext{
${ }^{9}$ En términos generales, la adaptación ocurre cuando una forma gramatical (GI) deriva de una forma más gramatical (G2). La refuncionalización, en cambio, ocurre cuando una forma gramatical (GI) deriva de una forma sin significado gramatical o menos gramatical (Go).

${ }^{10}$ En realidad, como sostiene el propio Willis (2010, 2016), no se considera necesariamente que la refuncionalización contribuya a crear una nueva función en el sistema; lo importante es que exista una nueva función del rasgo opaco u obsoleto en el proceso. Por otro lado, la aparición de un determinado fenómeno puede ser el resultado de la refuncionalización y funcionalización, esto es, deberse a la refuncionalización en una variedad específica y a la adecuación funcional en otra variedad de la misma lengua.
} 
ha perdido parte de su contenido semántico original, se considera "basura" (en el sentido de que es una forma no normativa, subestándar y no general) y retoma una categoría existente resegmentada $(l o+$ cuál $>$ lo cuálo $)$. Además, y a diferencia de los procesos típicos de gramaticalización, la (re)funcionalización puede ser un cambio discontinuo o "un salto" (Giacalone Ramat y Hopper 1998, Smith 201 I), de ahí que su documentación sea abundante en una sincronía dada, especialmente en los siglos XX y XXI.

Con todo, creemos que la "nueva” partícula ¿lo cuálo?, como proceso de cambio gramatical (¿cuál? > ¡lo cuálo?), puede entenderse mejor como un caso de (re)funcionalización. Tal y como se ha presentado previamente, la (re)funcionalización implica el desarrollo de una nueva función que, en este caso, sería sintáctico-pragmática. Un elemento pierde parte de su función original (pronombre) y pasa a emplearse para codificar una nueva categoría discursiva (partícula) con otros fines comunicativos, interrogativos, y con matiz irónico y jocoso. ¿Lo cuálo? representa, por tanto, un ejemplo más de creación (formación de un nuevo elemento gramatical o partícula) y reutilización de material gramatical previo para nuevos propósitos: preguntar por algo identificable, mencionado previamente o sobrentendido, mostrando a la vez sorpresa, extrañeza o ironía; construcción que se debe asimilar primero en el vocabulario como punto de partida para la (re)funcionalización. En este proceso es importante matizar que la construcción (re)funcionalizada muestra novedad conceptual ('‘cómo, qué?'), esto es, el hablante analiza una propiedad semántica que se encuentra en el contexto como una propiedad inherente de la unidad léxica (Croft 2000) ${ }^{11}$.

Para terminar, hay que recordar que las razones de los procesos de gramaticalización deben buscarse en las necesidades comunicativas de los hablantes, la mayoría de las veces por una tendencia a ser concisos y ganar claridad en la expresión, pero también por otras intenciones, irónicas y jocosas, como el caso que hemos revisado.

\section{Conclusiones}

En aragonés, judeoespañol y asturiano el pronombre cual/cuál distingue el género y el número. Este modelo ha podido servir, en unas áreas peninsulares y en otras, de base para la reestructuración del sistema del español, que se produce cuando los hablantes dotan a la forma cual/cuál de marcas de género y número, siguiendo para ello

\footnotetext{
${ }^{11}$ La refuncionalización es distinta de la gramaticalización en la medida en que, en este último proceso, un elemento léxico adquiere una función gramatical y sufre cambios formales dentro de este proceso, mientras que, en el primero, se conserva una función gramatical antigua y desvanecida a la cual se le asigna una nueva función (Vincent I995). Ambos fenómenos producen un enriquecimiento de las herramientas discursivas, ya que se crean nuevos valores textuales dentro de nuevos contextos.
} 
el patrón de otras formas pronominales como cuyo (cuya, cuyos, cuyas). Para García de Diego (I990 [1918]: 247), por ejemplo, de la forma cual se habría creado el femenino cuala, y de esta forma femenina el masculino cualo.

En este trabajo se ha propuesto que ¿lo cuálo? es una "nueva" partícula funcionalizada, propia del registro coloquial, que depende del contexto de uso y de la intención del hablante. Su uso estaría restringido al español europeo, aunque se puede documentar también de manera esporádica en algunas variedades del español en América. Desde el punto de vista normativo, la Academia "recomienda evitar todas estas formas en cualquier registro lingüístico porque están muy desprestigiadas" (RAE y ASALE 2009: I 559). De hecho, no solo estarían desprestigiadas, como formas vulgares que son, sino que "marcan a quien las utiliza" (Instituto Cervantes 20I2: 252). Según los datos del español actual analizados, procedentes del Corpus del español: Web/Dialectos y de la red social Twitter, parece que los usuarios harían con frecuencia caso omiso de estas recomendaciones.

Del mismo modo que sucede con el relativo elllallo cual, que puede funcionar como conector discursivo cuando aparece encabezando una oración yuxtapuesta, pues conforma un enunciado independiente del que contiene el antecedente (Brucart 1999), ¿lo cuálo? se convierte también en partícula —en el sentido de que puede guiar la interpretación del discurso - cuando aparece, sobre todo, en oraciones independientes para asociarse a contenidos expresados con anterioridad en el discurso. Esta "nueva" partícula ha podido seguir un proceso de funcionalización, en el sentido de que presenta un nuevo significado ('cómo, (el) qué, qué cosa') y una nueva función en el sistema gramatical del español.

\section{REFERENCIAS BIBLIOGRÁFICAS}

ALLA $200 \mathrm{I}$ = ACADEMIA DE LA LLINGUA ASTURIANA (200I): Gramática de la llingua asturiana, $3 .^{\mathrm{a}} \mathrm{ed}$., Uviéu: Academia de la Llingua Asturiana.

ALPI 1962 = Atlas Lingüistico de la Peninsula Ibérica. I. Fonética, Madrid: Consejo Superior de Investigaciones Científicas.

AlVAR, Manuel (1950): El habla del Campo de Jaca, Salamanca: Universidad de Salamanca.

AlVAR, Manuel (1953): El dialecto aragonés, Madrid: Gredos.

AlVAR, Manuel y Bernard POTTIER (I983): Morfología histórica del español, Madrid: Gredos.

BORAO, Jerónimo (I 884): Diccionario de voces aragonesas, Zaragoza: Diputación Provincial.

BRIZ, Antonio (1998): El español coloquial en la conversación. Esbozo de pragmagramática, Barcelona: Ariel.

BRIZ, Antonio y Grupo Val.Es.Co (2008): “Diccionario de partículas discursivas del español”, en

Dolores Azorín Fernández et al. (eds.), Actas del II Congreso Internacional de Lexicografía Hispánica. 
El diccionario como puente entre las lenguas y culturas del mundo, Alicante: Biblioteca Virtual Miguel de Cervantes, pp. 217-227.

BRIZ, Antonio, Salvador PONS y José PORTOLÉS (coords.) (2008): Diccionario de partículas discursivas del español. http://www.dpde.es.

BRUCART, José M. (I999): “La estructura del sintagma nominal: las oraciones de relativo”, en Ignacio Bosque y Violeta Demonte (dirs.), Gramática descriptiva de la lengua española, Madrid: Espasa Calpe, vol. I, pp. 395-522.

Corominas, Joan y José Antonio PASCUAL (1980-I99i): Diccionario crítico etimológico castellano e hispánico, Madrid: Gredos. 6 vols.

CROFT, William (2000): Explaining Language Change: An Evolutionary Approach, Harlow: Longman.

DAvies, Mark (2016): Corpus del español: Web/Dialectos, Provo: Brigham Young University. http://www.corpuesdelespanol.org.

DíAZ GonZÁlez, Olga J. (I986): El habla de Candamo (aspectos morfosintácticos y vocabulario), Oviedo: Universidad de Oviedo.

DíAZ-MAS, Paloma (I983): "El sincretismo lingüístico-cultural sefardí a la luz de dos textos aljamiados”, Revista de Filología Románica, I, pp. 225-236.

FERNÁNDEZ-ORDÓNEZZ, Inés (dir.) (2005-2019): Corpus oral y sonoro del español rural, Madrid: Universidad Autónoma de Madrid. http://www.corpusrural.es.

GARCÍA DE DiegO, Vicente (I 990 [1 9 I 8]): “Caracteres fundamentales del dialecto aragonés”, Archivo de Filología Aragonesa, 44-45, pp. 23 I-254.

GarCía Moreno, Aitor (2004): Relatos del pueblo ladinán: Méam Lo'ez de Éxodo, Madrid: Consejo Superio de Investigaciones Científicas.

Giacalone Ramat, Anna y Paul J. Hopper (i998): "Introduction", en Anna Giacalone Ramat y Paul J. Hopper (eds.), The Limits of Grammaticalization, Amsterdam/Philadelphia: John Benjamins, pP. I-I I.

GÓmEZ ORTín, Francisco (2004): "El dialecto murciano y sus variedades”, Tonos Digital, 8, pp. 7-27.

GÓMEZ SÁNCHEZ, Dionisia (20 I I): "El habla y las tradiciones populares de Fuente-Álamo”, Al-Basit. Revista de estudios albacetenses, 56, pp. 223-255.

Harris, Alice C. y Lyle CAmpbell (I995): Historical Syntax in Cross-Linguistic Perspective, Cambridge: Cambridge University Press.

HeInE, Bernd (2003): “On degrammaticalization”, en Barry J. Blake y Kate Burridge (eds.), Historical Linguistics 200I: Selected Papers from the ISth International Conference on Historical Linguistics, Amsterdam/Philadelphia: John Benjamins, pp. I63-I79. https://doi.org/I0.1075/cilt.237.I 2hei.

INSTITUTO CERVANTES (20 I 2): El libro del español correcto. Claves para escribir y hablar bien en español, Madrid: Instituto Cervantes/ Espasa.

KaltenbÖCK, Gunther, Bernd HeIne y Tania KuteVa (20 I I): "On thetical grammar", Studies in Language, 35 (4), pp. 852-897. https://doi.org/10.1075/sl.35.4.03 kal.

LASS, Roger (I990): "How to do things with junk: Exaptation in language evolution”, Journal of Linguistics, 26, pp. 79-102. https://doi.org/I0.1017/s00222267000I 4432.

LÓPEZ NAVARRETE, Rafael y José M. TORRES BELMONTE (2008): El habla de los pueblos turolenses de la comarca Gúdar, Madrid: Visión. 
MARÍN I RAMOS, Ferrán (2009): Reflexiones en torno al aragonés de Luna: pasado y presente de una lengua, Luna (Zaragoza): Asociación Cultural "Banzo Azcón”.

METZeLtin, Michael (2009): "El aragonés entre las lenguas románicas”, II Congreso de l'Aragonés. Actas, Zaragoza: l'Academia de l'Aragonés, pp. I37-I 56.

NAGORE LAín, Francho (1989): Gramática de la lengua aragonesa, Zaragoza: Mira.

NoGuÉs, Romualdo (I 898): Cuentos, tipos y modismos de Aragón, Madrid: Fernando Fe Librero.

Norde, Muriel (2009): Degrammaticalization, Oxford: Oxford University Press.

NúNEZZ MÉNDEZ, Eva (20I 5): “¿Retorno lingüístico? El judeoespañol y su diacronismo”, Retorno, $\mathrm{I}(\mathrm{I})$, pp. 89-I 32.

PATO, Enrique y Carlota DE BENITO (2017): “Tráenolos para comérnolos o la 'transposición' del clítico en español actual”, Philologica Jassyensia, I3 (I), pp. I 2 I-I 36.

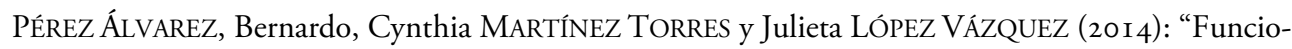
namiento discursivo del relativo artículo + cual", Signos Lingüisticos, Io(19), pp. 38-65.

PONS, Salvador (1998): Conexión y conectores. Estudio de su relación en el registro informal de la lengua, Valencia: Universidad de Valencia. Anejo XXVII de la revista Cuadernos de Filología.

PORTOLÉS, José (1998): Marcadores del discurso, Barcelona: Ariel.

QUINTANA, Aldina (200I): “Concomitancias lingüísticas entre el aragonés y el ladino (judeoespañol)”, Archivo de Filología Aragonesa, 57-58, pp. I63-192.

REAl ACADEMIA ESPAÑola: Banco de datos CORDE. Corpus diacrónico del español, Madrid: RAE. http://www.rae.es.

ReAl ACADEMia EsPañola: Banco de datos CREA. Corpus de referencia del español actual, Madrid: RAE. http://www.rae.es.

RAE y ASALE 2005 = REAL ACADEMIA ESPAÑOLA y ASOCIACIÓN DE ACADEMIAS DE LA LENGUA ESPAÑOLA (2005): Diccionario panhispánico de dudas, Madrid: Santillana. http://www.rae.es.

RAE y ASALE 2009 = REAL ACADEMIA ESPAÑOLA y ASOCIACIÓN DE ACADEMIAS DE LA LENGUA ESPAÑOla (2009): Nueva gramática de la lengua española, Madrid: Espasa. http://www.rae.es.

SAROÏHANDY, Jean-Joseph (I898): "Le dialecte de Graus", Annuaire de l'École Pratique des Hautes Études París, pp. 85-95.

SAROÏHANDY, Jean-Joseph (I90I): "Mission de M. Saroïhandy en Espagne”, Annuaire de l'École Pratique des Hautes Études París, pp. I06-i I 8.

SECO, Manuel (1986): Diccionario de dudas y dificultades de la lengua española, Madrid: Espasa-Calpe.

SELFA SASTRE, Moisés (2005-2006): "Estudio fonético-fonológico y morfosintáctico del habla del Valle Medio del Ésera (Huesca)", Archivo de Filología Aragonesa, 61-62, pp. I65-I 84.

SMITH, John Charles (201 I): "Change and continuity in form-function relationships", en Martin Maiden, John Charles Smith y Adam Ledgeway (eds.), The Cambridge History of the Romance Languages, Volume I, Structures, Cambridge: Cambrigde University Press, pp. 269-3 I7.

TOMEY, Julio Víctor (I90I): Cosas baturras, Barcelona: Maucci.

TraUgOTT, Elizabeth C. (2004): “Exaptation and grammaticalization”, en Minoji Akimoto (ed.), Linguistic Studies Based on Corpora, Tokyo: Hituzi Syobo, pp. I33-I 56. 
UMPhrey, George W. (1987 [r91 r]): "El dialecto aragonés”, Archivo de Filología Aragonesa, 39, pp. I63-20I.

VAN DIJK, Teun A. y Walter KINTSCH (I983): Strategies of discourse comprehension, New York: Academic Press.

VESPERTINO RODRÍGUEZ, Antonio (2002-2004): "El aragonés de la literatura aljamiado-morisca”, Archivo de Filología Aragonesa, 59-60, pp. I73 I-1754.

VINCENT, Nigel (1995): “Exaptation and grammaticalization”, en Henning Andersen (ed.), Historical Linguistics I993: Selected Papers from the II ${ }^{\text {th }}$ International Conference on Historical Linguistics, Amsterdam/Philadelphia: John Benjamins, pp. 433-445. https://doi.org/I o. I075/cilt. I 24.34vin.

WaGneR, Max Leopold (I9I4): Beiträge zur Kenntnis des Judenspanischen von Konstantinopel, Wien: Hölder.

WILLIS, David (2010): "Degrammaticalization and obsolescent morphology: Evidence from Slavonic", en Ekaterini Stathi, Elke Gehweiler y Ekkehard König (eds.), Grammaticalization: Current Views and Issues, Amsterdam/Philadelphia: John Benjamins, pp. I 5 I-I 78. https://doi.org/I0.1075/slcs. I I9.09wil.

WILLIS, David (2016): "Exaptation and degrammaticalization within an acquisition-based model of abductive reanalysis", en Muriel Norde y Freek Van de Velde (eds.), Exaptation in Language Change, Amsterdam/Philadelphia: John Benjamins, pp. 197-225. https://doi.org/I0.1075/cilt.336.07wil.

ZAMOra Vicente, Alonso (1967): Dialectología española, Madrid: Gredos. 